\title{
REGENERAÇÃO NATURAL EM NASCENTES DEGRADADAS
}

Auwdreia Pereira Alvarenga' (in memoriam), Israel Marinho Pereira ${ }^{2}$, Soraya Alvarenga Botelho ${ }^{3}$, Daniela Torres Morandi ${ }^{4}$, Fillipe Vieira de Araújo ${ }^{5}$

1 Engenheira florestal, Universidade Federal dos Vales do Jequitinhonha e Mucuri, Diamantina, Brasil.

2 Professor do curso de Engenharia florestal, Universidade Federal dos Vales do Jequitinhonha e Mucuri, Diamantina, Brasil.

3 Professora do curso de Engenharia florestal, Universidade Federal de Lavras, Lavras, Brasil.

4 Graduanda de Engenharia florestal, Universidade Federal dos Vales do Jequitinhonha e Mucuri, Diamantina (dtmorandi@gmail.com), Brasil.

5 Doutorando do Programa de Pós-Graduação em Ciência Florestal, Universidade

Federal dos Vales do Jequitinhonha e Mucuri, Diamantina, Brasil.

Recebido em: 08/04/2016 - Aprovado em: 30/05/2016 - Publicado em: 20/06/2016 DOI: 10.18677/Enciclopedia_Biosfera_2016_098

\begin{abstract}
RESUMO
A condução do processo de regeneração natural tem demonstrado ser uma técnica viável para restauração de ecossistemas em ambientes ciliares. Na busca por métodos e técnicas de aplicação de projetos de restauração ambiental se insere o presente estudo. Este trabalho faz um inventário florístico da regeneração natural e avalia como o estrato regenerante pode funcionar como um bom indicador ecológico de restauração natural de um ecossistema. O objetivo é fazer um levantamento florístico e estrutural das comunidades regenerante e arbóreo-arbustiva adulta em uma área de mata ciliar no entorno de uma nascente. Os resultados mostram que a comparação entre a estrutura e fitossociologia de comunidades entre estratos regenerantes e adultos pode ser um bom indicativo de como se dá o processo de regeneração natural em um ecossistema. $O$ trabalho foi desenvolvido na bacia do rio Capivari, Itumirim MG. As conclusões apontam para a descoberta de espécies de importância potencial para serem utilizadas na restauração de nascentes onde haja necessidade do plantio além de fornecer informações que podem auxiliar na tomada de decisões conservacionistas e dados ecológicos sobre o funcionamento da floresta em áreas de nascentes com diferentes graus de impacto ou degradação.
\end{abstract}

PALAVRAS-CHAVE: Florística, restauração, sucessão ecológica.

\section{NATURAL REGENERATION AS INDICATIVE OF THE RESTORATION OF ECOSYSTEM FUNCTIONS IN DEGRADED SPRINGS}

\begin{abstract}
In the search for methods and application techniques of degraded areas recovery projects fall within this study. This work is a floristic survey of natural regeneration and assesses how the refined layer can act as a good environmental indicator of natural restoration of an ecosystem. The goal is to make a floristic and structural survey of regenerating and mature trees and shrubs communities in a riparian area in ENCICLOPÉDIA BIOSFERA, Centro Científico Conhecer - Goiânia, v.13 n.23; p. 1130 2016
\end{abstract}


the vicinity of a spring. The results show that the comparison between the structural and phytosociological communities between woody strata and adults can be a good indication of how is the natural regeneration process in an ecosystem. The study was developed in the basin of Capivari river in the southern region of Minas Gerais. The findings point to the potential importance of species discovery for use in restoring springs where there is need for planting and provides information that can assist in making conservation decisions and ecological data on forest dynamics operating in areas with springs varying degrees of shock or degradation.

KEYWORDS: ecological succession, recovery of degraded areas, floristic

\section{INTRODUÇÃO}

O aspecto mais importante da restauração florestal é a possibilidade do reestabelecimento das funções do ecossistema em ambientes prioritários como as matas ciliares. A vegetação no entorno de rios, lagoas, nascentes cumpre inúmeras funções ecológicas e serviços ecossistêmicos como impedir ou controlar a erosão das vertentes, atuar como corredores ecológicos para dispersão de propágulos de plantas e movimentação de fauna, além de ajudarem a controlar o volume e a vazão da água, o que é imprescindível na qualidade ambiental de toda uma bacia hidrográfica (MALLMANN et al., 2016).

Dentre as metodologias aplicadas a restauração em matas ciliares, a condução da regeneração natural (somada à remoção das fontes de degradação), é a mais utilizada em áreas com capacidade de rebrota e com a presença de remanescentes florestais circunvizinhos que irão fornecer propágulos vegetativos (RODRIGUES et al., 2009; SILVA et al., 2016).

O estudo da regeneração natural envolvendo as análises de dinâmicas é importante para a compreensão da mudança ecológica sucessional das espécies em um fragmento, sendo essa sucessão uma ferramenta, que possibilita entender como as comunidades biológicas se regeneram e sobrevivem em uma paisagem cada vez mais fragmentada (LOPES et al., 2016).

A implantação de projetos de restauração florestal em matas ciliares requer grande quantidade de recursos humanos e financeiros, além da possibilidade de prever sobre o futuro do projeto com relação á diferentes técnicas que venham a ser implantadas (CASTRO et al., 2013).

Com base nessas questões alguns trabalhos têm demonstrado que a melhor técnica a ser utilizada para restauração de ecossistemas em ambientes ciliares é a condução do processo de regeneração natural das funções do ecossistema (SOUZA et al, 2012), o que torna o processo menos custoso e mais eficiente. Por isso o desenvolvimento de trabalhos sobre regeneração natural das matas ciliares em muito pode ajudar na conservação dos cursos d'água e manutenção dos processos geossistêmicos em bacias hidrográficas, além de fornecer informações importantes para a elaboração e implantação de planos de manejo e projetos de restauração nessas áreas (GONÇALVES, 2015).

Segundo ALMAZÁN-NÚÑEZ (2012) o entendimento do procedimento de regeneração prescinde de levantamentos florísticos que subsidiarão estudos sobre riqueza, biogeografia, diversidade e estrutura das florestas, pois a forma com que a vegetação se regenera está intrinsecamente relacionada a tais fatores ecológicos.Diante do exposto, o objetivo geral desse estudo foi avaliar a capacidade de restauração da vegetação ciliar no entorno de uma nascente degradada por meio da análise estrutural do estrato regenerante.. 


\section{MATERIAL E MÉTODOS}

Os experimentos foram conduzidos em duas nascentes. Uma das nascentes, localiza-se no sítio Grupiara, que pertence à bacia hidrográfica do Rio Capivari, afluente do Rio Grande, margem esquerda, e está entre as coordenadas geográficas $21^{\circ} 14^{\prime} 07,2$ ' de latitude sul e 44ㅇ 52' 44,7' de longitude oeste, a 838 metros de altitude, no município de Lavras, MG. A outra nascente encontra-se localizada no sítio Perobas, no município de Itumirim, MG, pertence à bacia do Rio Capivari, margem direita, e encontra-se entre as coordenadas $21^{\circ} 16^{\prime} 41,7$ " de latitude sul e $44^{\circ} 52$ '15, 6 " de longitude oeste, a 892 metros de altitude.

A nascente situada no sítio Grupiara foi denominada de Nascente 1, classificada quanto ao estado de conservação, como uma área perturbada, ou seja, não apresentou 50 metros de vegetação em seu entorno, mas apresentou bom estado de conservação, apesar de estar ocupada por gramíneas exóticas. A nascente situada no sítio Perobas (Nascente 2), foi decomposta em duas áreas, devido ao fato de que um lado desta nascente foi classificado como perturbado (Área perturbada) e o outro como degradado (Área degradada), apresentando pouca vegetação, presença de bovinos e voçorocas. Ambas nascentes foram cercadas num raio de 50 metros (FERREIRA et al., 2009).

De acordo com CARVALHO et al. (2005), o clima da região é do tipo Cfa de Köppen (úmido subtropical); a temperatura média anual é de $16,7^{\circ} \mathrm{C}$, com médias mensais variando de $13,0^{\circ} \mathrm{C}$, em julho a $19,7^{\circ} \mathrm{C}$, em janeiro; a precipitação média anual é de $2.108 \mathrm{~mm}$, com médias mensais variando de $341 \mathrm{~mm}$, no trimestre mais chuvoso (dezembro a fevereiro) a $35 \mathrm{~mm}$, no trimestre mais seco (junho a agosto). Entre os solos da região de estudo predominam os Latossolos Amarelos Distróficos típicos e Latossolos Vermelhos-Amarelos Distróficos típicos.

O inventário florístico do estrato arbóreo foi realizado em março de 2003, onde foram amostrados os indivíduos arbóreos vivos com DAP (diâmetro à altura do peito) $\geq 5 \mathrm{~cm}$. Os indivíduos, que não foram identificados no campo, foram coletados para identificação botânica, pela comparação com exsicatas existentes no Herbário da Universidade Federal de Lavras (Herbário ESAL), consulta à literatura clássica taxonômica e a especialistas da UFLA (SOUZA, et al., 2012).

Para a avaliação florística da regeneração natural e do estrato arbóreo, na área aberta em restauração, foram demarcadas, na nascente 1, 37 parcelas de $10 \mathrm{x}$ $2 \mathrm{~m}\left(20 \mathrm{~m}^{2}\right)$, com uma área amostral de $740 \mathrm{~m}^{2}$. Na nascente 2, foram demarcadas 74 parcelas de $10 \times 2 \mathrm{~m}\left(20 \mathrm{~m}^{2}\right)$, sendo 37 parcelas na área perturbada e 37 na área degradada, totalizando uma área amostral de $1480 \mathrm{~m}^{2}$. Considerou-se como regeneração natural $(\mathrm{RN})$ todos os indivíduos arbóreos com DAP inferior a $5 \mathrm{~cm}$ e altura superior a $10 \mathrm{~cm}$. No sub-bosque do remanescente, foi realizada somente uma coleta, em maio de 2003. Já o levantamento florístico da RN, na área aberta em restauração, foi realizado em maio e em dezembro de 2003, quando todos os indivíduos, com altura maior que $10 \mathrm{~cm}$ e DAP menor que $5 \mathrm{~cm}$.

Foram estimados, para a RN e para o estrato arbóreo, os parâmetros fitossociológicos básicos, como: frequência, densidade e classes de tamanho da RN (Classe $1=<30 \mathrm{~cm}$; Classe $2=30$ a $150 \mathrm{~cm}$; Classe $3=150$ a $300 \mathrm{~cm}$; Classe $4=$ $>300 \mathrm{~cm}$ ), em valores absolutos e relativos e índice de RN. Para determinação da diversidade florística, foi utilizado o índice de Shannon-Weaver $\left(H^{\prime}\right)$ e de equabilidade de Pielou (J'), calculados tanto para a regeneração quanto para a vegetação arbustivo-arbórea. Para a comparação da RN entre as nascentes e a 
vegetação arbustivo-arbórea, foi utilizado o índice de similaridade de Jaccard (SJ) (FERREIRA et al., 2009)..

\section{RESULTADOS E DISCUSSÃO}

No levantamento florístico do estrato arbóreo foram registrados um total de 218 indivíduos pertencentes a 21 famílias, 41 gêneros e 51 espécies (Quadro 1). Na nascente 1 foram identificados 148 indivíduos pertencentes a 20 famílias, 29 gêneros e 37 espécies. As famílias que apresentaram o maior número de espécies foram Fabaceae Faboideae, Lauraceae, Anacardiaceae, Meliaceae, Annonaceae, Flacourtiaceae e Myrtaceae. Entre os gêneros com maior número de espécies, destacam-se Machaerium, Ocotea, Rollinea, Casearia e Nectandra. Estes gêneros contribuíram com $41,4 \%$ das espécies.

TABELA 1- Relação das espécies registradas nas matas ciliares no entorno de duas nascentes na região sul de Minas Gerais, MG, dispostas em ordem alfabética de família, seguidas do número de indivíduos. Em que: GE = grupo ecológico $(P=$ pioneira; $C L=$ clímax exigente de luz e $C S=$ clímax tolerante à sombra); $\mathrm{SD}=$ síndrome de dispersão (zoo = zoocórica; ane = anemocórica e aut = autocórica); N1 = nascente 1 e N2 = nascente 2; $\mathrm{EA}=$ estrato arbóreo, $\mathrm{RSB}$ = regeneração do sub-bosque e RAA= regeneração na área alterada.

\begin{tabular}{|c|c|c|c|c|c|c|c|c|c|c|}
\hline \multirow[b]{2}{*}{ Família/Espécie } & \multirow[b]{2}{*}{$\mathrm{GE}^{\star}$} & \multirow[b]{2}{*}{ SD } & \multicolumn{3}{|c|}{ EA } & \multicolumn{2}{|c|}{ RSB } & \multicolumn{2}{|c|}{ RAA } & \multirow{2}{*}{$\begin{array}{c}\text { Tota } \\
\text { I }\end{array}$} \\
\hline & & & N1 & N2 & $\begin{array}{c}\text { Tota } \\
\text { I }\end{array}$ & N1 & N2 & N1 & N2 & \\
\hline Anacardiaceae & & & & & & & & & & \\
\hline Lithraea molleoides (Vell.) Engler & $P$ & $z 00$ & 52 & 1 & 53 & 6 & 0 & 10 & 1 & 17 \\
\hline Schinus terebinthifolius Raddi & $P$ & zoo & 2 & 0 & 2 & 3 & 0 & 0 & 0 & 3 \\
\hline $\begin{array}{l}\text { Tapirira guianensis Aublet } \\
\text { Annonaceae }\end{array}$ & $P$ & $z 00$ & 1 & 0 & 1 & 2 & 1 & 1 & $\begin{array}{l}0 \\
0\end{array}$ & 4 \\
\hline Rollinia laurifolia Schltdl. & $\mathrm{CL}$ & $z 00$ & 1 & 0 & 1 & 0 & 4 & 0 & 0 & 4 \\
\hline $\begin{array}{l}\text { Rollinia sericea (R.E.Fries) R.E.Fries } \\
\text { Apocynaceae }\end{array}$ & $\mathrm{CL}$ & $z 00$ & 1 & 3 & 4 & 0 & 1 & 0 & $\begin{array}{l}0 \\
0\end{array}$ & 1 \\
\hline $\begin{array}{l}\text { Aspidosperma parvifolium A.DC. } \\
\text { Aquifoliaceae }\end{array}$ & $C L$ & ane & & & & 0 & 2 & 1 & $\begin{array}{l}1 \\
0\end{array}$ & 4 \\
\hline $\begin{array}{l}\text { Ilex cerasifolia Reissek } \\
\text { Araliaceae }\end{array}$ & CS & $z 00$ & & & & 1 & 0 & 0 & $\begin{array}{l}0 \\
0\end{array}$ & 1 \\
\hline $\begin{array}{l}\text { Dendropanax cuneatus (DC.) Decne } \\
\text { \& Planchon }\end{array}$ & CS & $z 00$ & 2 & 0 & 2 & 42 & 1 & 4 & 0 & 47 \\
\hline Asteraceae & & & & & & & & & 0 & \\
\hline Baccharis lymanii G.M.Barroso & $\mathrm{nc}$ & ane & & & & 0 & 0 & 31 & 42 & 73 \\
\hline $\begin{array}{l}\text { Gochnatia polymorpha (Less.) } \\
\text { Cabrera }\end{array}$ & $\mathrm{P}$ & ane & & & & 0 & 0 & 2 & 0 & 2 \\
\hline $\begin{array}{l}\text { Vernonanthura diffusa (Less.) } \\
\text { H.Robinson }\end{array}$ & $P$ & ane & & & & 0 & 1 & 0 & 0 & 1 \\
\hline $\begin{array}{l}\text { Vernonanthura phosphorica (Vell.) H. } \\
\text { Robinson }\end{array}$ & $P$ & ane & & & & 0 & 0 & 0 & 11 & 11 \\
\hline $\begin{array}{l}\text { Vernonia polyanthes Less. } \\
\text { Bignoniaceae }\end{array}$ & $P$ & ane & & & & 0 & 1 & 1 & $\begin{array}{c}38 \\
0\end{array}$ & 40 \\
\hline Tabebuia chrysotricha (Mart.) & $\mathrm{CL}$ & ane & & & & 0 & 0 & 1 & 0 & 1 \\
\hline
\end{tabular}


Standley

Tabebuia serratifolia (Vahl) Nichols

Boraginaceae

Cordia ecalyculata Vell.

CS ane

Cordia trichotoma (Vell.) Arrab.

Celtidaceae

Celtis iguanaea (Jacquin) Sargent

Ebenaceae

Diospyros inconstans Jacquin

Erythroxylaceae

Erythroxylum pelleterianum A.St-Hil.

Euphorbiaceae

Actinostemon concolor (Sprengel)

Müll.Arg.

Croton floribundus Sprengel

Croton urucurana Baillon

Sapium glandulosum (L.) Morong

Fabaceae Caesalpinioideae

Bauhinia forficata Link

Bauhinia longifolia (Bongard) Steudel

Copaifera langsdorffii Desf.

CS zoo

CL zoo

$\begin{array}{llll}\mathrm{P} & \text { zoo } & 1 & 11\end{array}$

$\begin{array}{llll}0 & 0 & 2 & 0\end{array}$

2

CL zoo

CS zoo

0

59

0

1

$\begin{array}{lllll}0 & 1 & 0 & 0 & 1 \\ & & & 0 & \end{array}$

$\begin{array}{lllll}2 & 3 & 6 & 0 & 11\end{array}$

CS

$P$ auto $2 \quad 0 \quad 2$

$P$ auto

$\begin{array}{lllll}C L & \text { zoo } & 0 & 1 & 1\end{array}$

Senna macranthera (Vell.) Irwin \&

Barneby

\section{Fabaceae Faboideae}

Lonchocarpus muehlbergianus

Hassler

Machaerium hirtum (Vell.) Stellfeld

Machaerium nictitans (Vell.) Benth.

Machaerium stipitatum (DC.) Vogel

Machaerium villosum Vogel

Platycyamus regnellii Benth.

Platypodium elegans Vogel

Fabaceae Mimosoideae

Acacia polyphylla DC.

Albizia polycephala (Benth.) Killip

Inga striata Benth.

Piptadenia gonoacantha (Mart.)

Macbr.

Flacourtiaceae

Casearia decandra Jacquin

Casearia lasiophylla Eichler

Casearia sylvestris Swartz

Xylosma ciliatifolium (Clos) Eichler

Lauraceae

Aniba firmula (Nees \& Mart.) Mez

Endlicheria paniculata (Sprengel)

Macbr.

Nectandra grandiflora Nees

Nectandra lanceolata Nees

$\begin{array}{lccccccccc}\mathrm{CL} & \text { auto } & 0 & 2 & 2 & 1 & 10 & 0 & 0 & 11 \\ \mathrm{CL} & \text { auto } & 1 & 0 & 1 & 1 & 0 & 0 & 0 & 1 \\ \mathrm{CS} & \text { zoo } & 0 & 1 & 1 & 8 & 4 & 0 & 0 & 12 \\ \mathrm{P} & \text { zoo } & 0 & 2 & 2 & 0 & 2 & 0 & 0 & 2\end{array}$

0

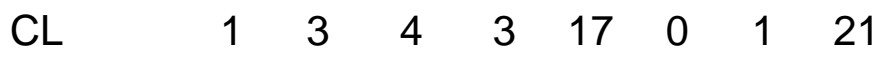

$\mathrm{CL}$ ane $\begin{array}{llllllll}7 & 3 & 10 & 7 & 4 & 17 & 2 & 30\end{array}$

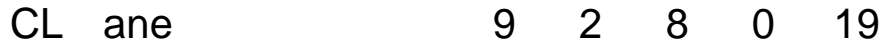

$\mathrm{CL}$ ane 200

$\mathrm{CL}$ ane $6 \begin{array}{llllllll}6 & 0 & 6 & 3 & 3 & 22 & 1 & 29\end{array}$

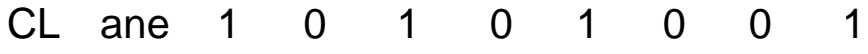

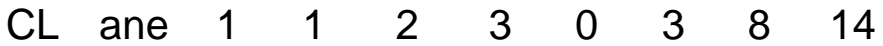

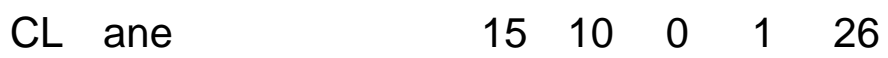

$\mathrm{CL}$ ane $\quad 3 \quad 0011004$

CL zoo $00 \begin{array}{llllllll} & 0 & 3 & 0 & 3 & 0 & 0 & 3\end{array}$

$\mathrm{P}$ auto $1 \quad 0 \quad 1$ 
Nectandra nitidula Nees

Nectandra oppositifolia Nees

Ocotea corymbosa (Meisner) Mez

Ocotea diospyrifolia (Meisner) Mez

Ocotea laxa (Nees) Mez

Persea pyrifolia Nees \& Mart.

Malpighiaceae

Heteropterys byrsonimifolia A.Juss.

Malvaceae

Ceiba speciosa (A.St.-Hil.) Gibbs \&

Semir

Guazuma ulmifolia Lam.

Luehea divaricata Mart. \& Zucc.

Luehea grandiflora Mart. \& Zucc.

Melastomataceae

Miconia brunnea Mart.

Miconia ibaguensis (Bonpl.) Triana

Meliaceae

Cedrela fissilis Vell.

Guarea kunthiana A.Juss.

Trichilia hirta L.

Monimiaceae

Mollinedia argyrogyna Perkins

Mollinedia widgrenii A.DC.

\section{Moraceae}

Ficus guaranitica Chodat

Maclura tinctoria (L.) D.Don.

Myrsinaceae

Myrsine coriacea (Sw.) Roem. \&

Schult.

Myrsine umbellata Mart.

Myrtaceae

Calyptranthes clusiifolia (Miq.)

O.Berg

Campomanesia pubescens (DC.)

O.Berg

Eugenia florida DC.

Gomidesia affinis (Cambess.)

D.Legrand

Myrcia fallax (Rich.) DC.

Myrcia rostrata DC.

Myrcia tomentosa (Aublet) DC.

Myrcia velutina O.Berg

Myrciaria floribunda (West) O.Berg

Psidium guineense $\mathrm{Sw}$.

Psidium guajava L.

Piperaceae

Piper aduncum L.

Rubiaceae
CL zoo

CS $z 00$

CL $z 00$

$\mathrm{CL} z 00$

CS zoo

CL zoo

P zoo

$\mathrm{CL}$ ane

$P \quad z o 0$

CL zoo

CL zoo

nc $z 00$

nc zoo

CL ane

CS zoo

nc zoo

CS $z 00$

CS zoo

$\begin{array}{lllll}n c & z 00 & 0 & 3 & 3\end{array}$

$\begin{array}{lllll}\mathrm{P} & \mathrm{z} 00 & 4 & 4 & 8\end{array}$

nc $\quad$ zoo

CL zoo

CS zoo

nc $z 00$

CS zoo

CL zoo

CL $z 00$

CL $z 00$

CL zoo

nc $z 00$

CS zoo

nc $z 00$

CL zoo

P $\quad$ zoo

$\begin{array}{ll}0 & 1 \\ 0 & 1\end{array}$ $\begin{array}{lllll}5 & 0 & 7 & 0 & 12\end{array}$

$\begin{array}{lllll}4 & 2 & 0 & 0 & 6\end{array}$

$\begin{array}{llllllll}1 & 0 & 1 & 1 & 0 & 0 & 0 & 1\end{array}$

$\begin{array}{llllllll}2 & 0 & 2 & 13 & 0 & 0 & 0 & 13\end{array}$

$\begin{array}{cccccccc}16 & 0 & 16 & 3 & 1 & 1 & 0 & 5\end{array}$

$\begin{array}{lllll}0 & 2 & 0 & 0 & 2\end{array}$

0

$\begin{array}{llllllll}0 & 1 & 1 & 0 & 1 & 0 & 0 & 1 \\ 0 & 1 & 1 & 0 & 0 & 9 & 0 & 9 \\ 3 & 0 & 3 & 4 & 0 & 0 & 0 & 4 \\ 2 & 0 & 2 & 20 & 0 & 0 & 0 & 20 \\ & & & 33 & 0 & 0 & 0 & 33\end{array}$

$\begin{array}{lllll}2 & 0 & 0 & 0 & 2\end{array}$

0

0

0

$\begin{array}{lllll}3 & 10 & 0 & 2 & 15\end{array}$

0

1

$\begin{array}{llllllll}5 & 5 & 10 & 15 & 35 & 2 & 5 & 57\end{array}$

0

$2 \quad 0 \quad 0 \quad 002$

$\begin{array}{llllllll}2 & 0 & 2 & 2 & 0 & 0 & 0 & 2\end{array}$

$\begin{array}{lllll}4 & 0 & 0 & 0 & 4\end{array}$

$\begin{array}{lllll}1 & 0 & 0 & 0 & 1\end{array}$

$\begin{array}{lllll}0 & 1 & 0 & 0 & 1\end{array}$

$\begin{array}{lllll}0 & 0 & 2 & 0 & 2\end{array}$

$\begin{array}{lllll}1 & 1 & 2 & 0 & 4\end{array}$

$\begin{array}{lllll}2 & 0 & 1 & 0 & 3\end{array}$

$\begin{array}{lllll}4 & 0 & 0 & 0 & 4\end{array}$

$\begin{array}{lllll}0 & 0 & 0 & 1 & 1\end{array}$

$\begin{array}{lllll}0 & 0 & 3 & 5 & 8\end{array}$

0

$\begin{array}{lllll}37 & 58 & 11 & 36 & 142\end{array}$ 
Chomelia sericea Müll.Arg.

Coutarea hexandra (Jacquin)

K.Schum.

Psychotria carthagenensis Jacquin

Rutaceae

Esenbeckia leiocarpa Engler

Zanthoxylum rhoifolium Lam.

Zanthoxylum riedelianum Engl.

Sapindaceae

Diatenopteryx sorbifolia Radlk.

Matayba elaeagnoides Radlk.

Sapotaceae

Chrysophyllum marginatum Radlk.

Siparunaceae

Siparuna cujabana (Mart.) A.DC.

Solanaceae

Cestrum laevigatum Schltdl.

Solanum granuloso-leprosum Dunal

Solanum lycocarpum A.St.-Hil.

Styracaceae

Styrax ferrugineus Nees \& Mart.

Verbenaceae

Aegiphila sellowiana Cham.

Aloysia virgata (Ruiz \& Pavón)

A.Juss.

Total

Na nascente 2, foram amostrados 70 indivíduos, 17 famílias, 26 gêneros e 28 espécies. Oito famílias contribuíram com $67,8 \%$ do número total de espécies, com Fabaceae Caesalpinioideae, Fabaceae Faboideae e Lauraceae ocupando a primeira posição, com 10,7\%, cada uma, seguida de Euphorbiaceae, Flacourtiaceae, Malvaceae, Moraceae e Sapindaceae, com 7,1\%, cada uma. Os gêneros Casearia e Nectandra destacaram-se pelo maior número de espécies, duas cada uma, estando os demais 24 gêneros (92,3\%) representados por uma única espécie.

SILVA et al., 2016 avaliando o processo de RN após plantio de enriquecimento em uma área de preservação permanente degradada em Lavras, MG, encontrou no censo do estrato arbóreo as espécies: Eremanthus incanus, Guazuma ulmifolia, Luehea divaricata, Machaerium vilosum, Lithraea molleoides, Vernonanthura phosphorica, Psidium guajava, Schinus terebentifolius e Tapirira guianensis.. Segundo os autores, o resultado demonstra que parte da fonte de propágulos é proveniente do banco de sementes da própria APP fornecido pelo método de enriquecimento.

O número de espécies com um único indivíduo amostrado na nascente 1 , foi de 17 , o que equivale a $11,5 \%$ do total. Já na nascente 2, 13 espécies apresentaram um único indivíduo amostrado, correspondendo a $18,6 \%$ do total. Isto pode refletir o menor tamanho do fragmento da nascente 2. No levantamento florístico da $R N$ foram registrados, no período de monitoramento (maio-dezembro/2003), um total de 1208 indivíduos pertencentes a 32 famílias, 62 gêneros e 85 espécies. A relação das espécies amostradas nas duas avaliações, encontra-se Quadro 1. 
$\mathrm{Na}$ nascente 1, no sub-bosque do remanescente, foram registrados 376 indivíduos pertencentes a 24 famílias, 40 gêneros e 51 espécies. As famílias que apresentaram o maior número de espécies foram Lauraceae, Myrtaceae, Fabaceae Faboideae, Anacardiaceae, Fabaceae - Caesalpinioideae e Flacourtiaceae. Juntas, essas famílias contribuíram com, aproximadamente, $55 \%$ do total de espécies amostradas neste ambiente. Entre os gêneros, os com maior número de espécies foram Machaerium e Nectandra, Bauhinia, Casearia, Ocotea, Luehea, Miconia, Myrsine e Myrcia. Estes nove gêneros contribuíram com 39,2\% das espécies. Foram encontradas, na nascente 1, no sub-bosque do remanescente, 13 espécies com um único indivíduo amostrado, o que equivale a $3,45 \%$ do total. Estas espécies podem ser consideradas raras neste local.

No remanescente associado à nascente 2, foram identificados 239 indivíduos, 23 famílias, 32 gêneros e 40 espécies. As famílias que se destacaram, apresentando maior número de espécies foram Fabaceae Faboideae, Lauraceae e Flacourtiaceae, Fabaceae Caesalpinioidea, e Annnonaceae, Fabaceae Mimosoideae, Myrtaceae e Verbenaceae, as quais contribuíram com $60 \%$ do número total de espécies identificadas. Os gêneros Bauhinia, Machaerium, Casearia e Nectandra, Rollinia e Myrcia contemplaram, juntas, $40 \%$ do total das espécies amostradas.

LOPES et al., 2016, estudando a dinâmica da regeneração natural no subbosque de Pinus caribaea Morelet. var. caribaea na Reserva Biológica de Saltinho, Tamandaré - PE, encontraram no levantamento realizado em 2007296 indivíduos, pertencentes a 33 famílias, 43 gêneros e 54 espécies. No segundo levantamento realizado em 2012 foram registrados 333 indivíduos (aumento de 12,5\%), pertencentes a 30 famílias (redução de 9,09\%), 37 gêneros (redução de 13,95\%) e 45 espécies (redução de $16,67 \%$ do total de espécies).

Para a nascente 1 e para a nascente 2 AP e $A D$, na área aberta em restauração, foram realizadas duas avaliações (Quadro 2). Na nascente 1, foram amostrados, na primeira e na segunda avaliação, respectivamente, 193 e 208 indivíduos, 23 e 24 famílias, 31 e 32 gêneros e 37 e 38 espécies. As famílias Fabaceae Faboideae, Myrtaceae, Asteraceae, Flacourtiaceae, Anacardiaceae, Bignoniacea, Lauraceae e Malvaceae, apresentaram o maior número de espécies, contribuindo com $59,4 \%$ do total de espécies amostradas nesta nascente, na primeira avaliação e $57,9 \%$ na segunda.

$\mathrm{Na}$ nascente 2 AP, foram encontrados 135 e 151 indivíduos, 11 famílias, 14 gêneros e 16 espécies, na primeira e na segunda avaliação, respectivamente. As famílias que apresentaram maior número de espécies foram Fabaceae Faboideae, Asteraceae, Solanaceae e Verbenaceae, representando 56,25\% do total de espécies. Foram registrados, na primeira e na segunda avaliação, respectivamente, 200 e 234 indivíduos, 15 famílias, 18 e 20 gêneros e 21 e 24 espécies. As famílias Asteraceae, Myrtaceae, Rutaceae, Solanaceae e Verbenaceae representaram $52,4 \%$ do total das espécies amostradas nesta nascente na primeira avaliação. Já na segunda avaliação, as famílias que mais se destacaram em número de espécies, foram a Asteraceae, Fabaceae Faboideae, Flacourtiaceae, Myrtaceae, Rutaceae, Solanaceae e Verbenaceae, contribuindo com $66,67 \%$ das espécies.

No Quadro 2, apresentam-se os parâmetros fitossociológicos do estrato arbóreo na qual observa-se, para a nascente 1, uma densidade média de 3.895 indivíduo.ha ${ }^{-1}$. A espécie que se destacou, apresentando $1.368,42$ ind.ha $^{-1}$, foi Lithraea molleoides, representando cerca de $35 \%$ do total de indivíduos. Outras sete 
espécies, que se destacaram foram Persea pyrifolia, Casearia sylvestris, Machaerium hirtum, Nectandra oppositifolia, Machaerium villosum, Myrsine

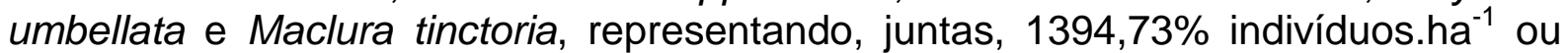
$35,81 \%$ do total. Dentre as 37 espécies amostradas na nascente 1, 17 são consideradas raras, uma vez que sua densidade relativa é menor que 1.

TABELA 2- Parâmetros fitossociológicos da RN da mata ciliar no entorno de uma nascente do rio Capivari em Itumirim, MG. Em que: $\mathrm{DR}$ = densidade relativa (\%); CRTR = classe relativa de tamanho da $\mathrm{RN}, \mathrm{FR}=$ freqüência relativa e $\mathrm{RN}=$ índice de RN.

\begin{tabular}{|c|c|c|c|c|c|c|c|c|}
\hline \multirow[t]{2}{*}{ Espécie } & \multicolumn{4}{|c|}{$\begin{array}{l}\text { Sub-bosque da } \\
\text { nascente } 1\end{array}$} & \multicolumn{4}{|c|}{$\begin{array}{l}\text { Área Alterada da } \\
\text { nascente } 1\end{array}$} \\
\hline & DR & $\mathbf{F R}$ & CRTR & $\mathbf{R N}$ & DR & & CRTR & RN \\
\hline Acacia glomerosa Benth & 3,99 & 3,70 & 4,88 & 4,19 & & & & \\
\hline $\begin{array}{l}\text { Albizia polycephala (Benth.) } \\
\text { Killip }\end{array}$ & 0,80 & 1,48 & 0,94 & 1,07 & 0,52 & 0,85 & 0,62 & 0,66 \\
\hline $\begin{array}{l}\text { Aloysia virgata (Ruiz \& Pavón) } \\
\text { A.Juss. }\end{array}$ & & & & & 3,63 & 2,54 & 1,51 & 2,56 \\
\hline $\begin{array}{l}\text { Aniba firmula (Nees \& Mart.) } \\
\text { Mez }\end{array}$ & 3,46 & 2,22 & 3,44 & 3,04 & & & & \\
\hline $\begin{array}{l}\text { Aspidosperma parvifolium } \\
\text { A.DC. }\end{array}$ & & & & & 0,52 & 0,85 & 0,62 & 0,66 \\
\hline Baccharis lymanii G.M.Barroso & & & & & 16,06 & 13,56 & 17,22 & 15,61 \\
\hline Bauhinia forficata Link & 0,27 & 0,74 & 0,37 & 0,46 & & & & \\
\hline $\begin{array}{l}\text { Bauhinia 1138ongifólia } \\
\text { (Bongard) Steudel }\end{array}$ & 0,27 & 0,74 & 0,11 & 0,37 & & & & \\
\hline $\begin{array}{l}\text { Calyptranthes clusiifolia (Miq.) } \\
\text { O.Berg }\end{array}$ & 0,53 & 0,74 & 0,74 & 0,67 & & & & \\
\hline $\begin{array}{l}\text { Campomanesia pubescens } \\
\text { (DC.) O.Berg }\end{array}$ & 0,53 & 0,74 & 0,74 & 0,67 & & & & \\
\hline Casearia decandra Jacquin & 0,27 & 0,74 & 0,37 & 0,46 & 0,52 & 0,85 & 0,62 & 0,66 \\
\hline $\begin{array}{l}\text { Casearia sylvestris Swartz } \\
\text { Ceiba speciosa (A.St.-Hil.) } \\
\text { Gibbs \& Semir }\end{array}$ & 2,13 & 2,96 & 2,10 & 2,40 & 7,25 & 5,93 & 7,08 & 6,75 \\
\hline $\begin{array}{l}\text { Celtis iguanaea (Jacquin) } \\
\text { Sargent }\end{array}$ & & & & & 3,63 & 4,24 & 3,85 & 3,90 \\
\hline Cestrum laevigatum Schltdl. & 0,80 & 1,48 & 0,59 & 0,96 & & & & \\
\hline Chomelia sericea Müll.Arg. & 5,32 & 2,96 & 5,97 & 4,75 & & & & \\
\hline $\begin{array}{l}\text { Chrysophyllum marginatum } \\
\text { (Hooker \& Arnot) Radlk. }\end{array}$ & & & & & 1,04 & 0,85 & 1,25 & 1,04 \\
\hline Copaifera langsdorffii Desf. & 2,13 & 2,22 & 1,76 & 2,04 & & & & \\
\hline Cordia ecalyculata Vell. & 0,27 & 0,74 & 0,04 & 0,35 & & & & \\
\hline $\begin{array}{l}\text { Cordia trichotoma (Vell.) Arrab. } \\
\text { Croton floribundus Sprengel }\end{array}$ & 0,80 & 0,74 & 0,59 & 0,71 & 0,52 & 0,85 & 0,62 & 0,66 \\
\hline Croton urucurana Baillon & & & & & 0,52 & 0,85 & 0,62 & 0,66 \\
\hline $\begin{array}{l}\text { Dendropanax cuneatus (DC.) } \\
\text { Decne \& Planchon }\end{array}$ & $\begin{array}{c}11,1 \\
7\end{array}$ & 5,19 & 11,54 & 9,30 & 2,07 & 0,85 & 1,97 & 1,63 \\
\hline Erythroxylum pelleterianum & 0,53 & 1,48 & 0,48 & 0,83 & 3,11 & 1,69 & 3,75 & 2,85 \\
\hline
\end{tabular}


A.St.-Hil.

Esenbeckia leiocarpa Engler

Eugenia florida DC.

$\begin{array}{llll}0,53 & 0,74 & 0,74 & 0,67\end{array}$

$\begin{array}{llll}1,06 & 1,48 & 1,22 & 1,26\end{array}$

Gochnatia polymorpha (Less.)

Cabrera

Gomidesia affinis (Cambess.)

D.Legrand

Guarea kunthiana A.Juss.

$\begin{array}{llll}0,27 & 0,74 & 0,37 & 0,46\end{array}$

Guazuma ulmifolia Lam.

Ilex cerasifolia Reissek

Lithraea molleoides (Vell.)

Engler

$\begin{array}{llll}0,53 & 1,48 & 0,74 & 0,92\end{array}$

Lonchocarpus muehlbergianus

Hassler

$\begin{array}{llll}0,27 & 0,74 & 0,37 & 0,46\end{array}$

$4,66 \quad 2,54 \quad 5,10 \quad 4,10$

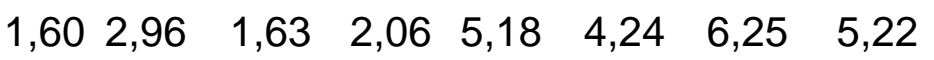

$\begin{array}{llll}0,80 & 1,48 & 0,94 & 1,07\end{array}$

Luehea divaricata Mart. \& Zucc. $0,27 \quad 0,74 \quad 0,11 \quad 0,37 \quad 1,04 \quad 1,69 \quad 1,25 \quad 1,33$

Luehea grandiflora Mart. \&

Zucc.

$1,06 \quad 1,48 \quad 1,49 \quad 1,34$

Machaerium hirtum (Vell.)

Stellfeld

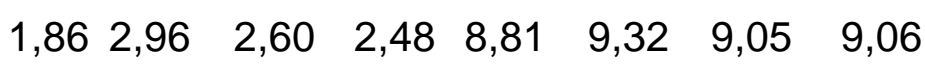

Machaerium nictitans (Vell.)

Benth.

Machaerium villosum Vogel

Maclura tinctoria (L.) D.Don.

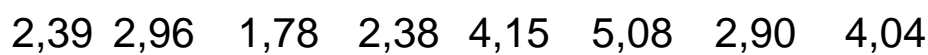

$\begin{array}{llllllll}0,80 & 2,22 & 0,68 & 1,23 & 11,40 & 13,56 & 9,03 & 11,33\end{array}$

Matayba elaeagnoides Radlk.

Miconia brunnea Mart.

$0,801,48$

$0,52 \quad 0,93$

$8,245,93$

$5,322,96$

$8,74 \quad 7,64$

$\begin{array}{llll}2,07 & 3,39 & 1,97 & 2,48\end{array}$

Miconia ibaguensis (Bonpl)

Triana

$\begin{array}{llll}8,78 & 5,93 & 10,69 & 8,46\end{array}$

Mollinedia argyrogyna Perkins

Myrcia rostrata DC.

$\begin{array}{llll}0,27 & 0,74 & 0,11 & 0,37\end{array}$

Myrcia tomentosa (Aublet) DC.

Myrcia velutina O.Berg

Myrciaria floribunda (West)

O.Berg

Myrsine ferruginea Spreng.

Myrsine umbellata Mart.

Nectandra lanceolata Nees

Nectandra nitidula Nees

Nectandra oppositifolia Nees

Ocotea corymbosa (Meisner)

Mez

Ocotea laxa (Nees) Mez

$\begin{array}{lll}0,27 & 0,74\end{array}$

$\begin{array}{lllll}0,04 & 0,85 & 0,68 & 0,85\end{array}$

$\begin{array}{llllllll}0,53 & 1,48 & 0,48 & 0,83 & 0,52 & 0,85 & 0,62 & 0,66\end{array}$

$\begin{array}{llll}1,06 & 1,48 & 1,22 & 1,26\end{array}$

$\begin{array}{llll}0,27 & 0,74 & 0,37 & 0,46\end{array}$

$3,992,96$

$2,913,29$

$5,854,44$

$5,57 \quad 5,29$

1,33 2,22

$1,34 \quad 1,63$

$1,061,48$

$0,97 \quad 1,17$

$\begin{array}{llll}0,27 & 0,74 & 0,11 & 0,37\end{array}$

Persea pyrifolia Nees \& Mart.

$3,463,70$

$2,66 \quad 3,27$

$0,80 \quad 0,74$

$0,94 \quad 0,83$

0,52

0,85

0,62

0,66

Piper aduncum $\mathrm{L}$.

$9,84 \quad 6,67$

8,94

$8,48 \quad 5,70$

$3,39 \quad 6,87$

5,32

Platypodium elegans Vogel

Psidium guajava L.

$0,80 \quad 1,48$

$0,59 \quad 0,96$

$\begin{array}{llll}1,55 & 2,54 & 1,30 & 1,80\end{array}$

$\begin{array}{llll}1,55 & 2,54 & 1,87 & 1,99\end{array}$

Psychotria carthagenensis Jacquin

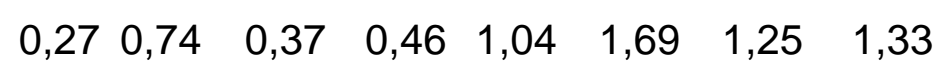




\begin{tabular}{|c|c|c|c|c|c|c|c|}
\hline Schinus terebinthifolius Raddi & $0,801,48$ & 0,85 & 1,04 & & & & \\
\hline $\begin{array}{l}\text { Siparuna cujabana (Mart.) } \\
\text { A.DC. }\end{array}$ & $0,27 \quad 0,74$ & 0,37 & 0,46 & & & & \\
\hline $\begin{array}{l}\text { Styrax ferrugineus Nees \& } \\
\text { Mart. }\end{array}$ & & & & 0,52 & 0,85 & 0,62 & 0,66 \\
\hline $\begin{array}{l}\text { Handroanthus chrysotrichus } \\
\text { (Mart. ex DC.) Mattos. }\end{array}$ & & & & 0,52 & 0,85 & 0,62 & 0,66 \\
\hline $\begin{array}{l}\text { Handroanthus serratifolius } \\
\text { (Vahl) S. O. Grose }\end{array}$ & & & & 1,04 & 1,69 & 0,73 & 1,15 \\
\hline Tapirira guianensis Aublet & $0,531,48$ & 0,08 & 0,70 & 0,52 & 0,85 & 0,62 & 0,66 \\
\hline Vernonia polyanthes Less. & & & & 0,52 & 0,85 & 0,62 & 0,66 \\
\hline $\begin{array}{l}\text { Xylosma ciliatifolium (Clos) } \\
\text { Eichler }\end{array}$ & $0,530,74$ & 0,74 & 0,67 & 1,04 & 0,85 & 1,25 & 1,04 \\
\hline Zanthoxylum rhoifolium Lam. & & & & 0,52 & 0,85 & 0,05 & 0,47 \\
\hline Total & 100100 & 100 & 100 & 100 & 100 & 100 & 100 \\
\hline
\end{tabular}

As espécies de maior freqüência relativa, ou seja, com melhor distribuição na nascente 1, foram Lithraea molleoides, Casearia sylvestris, Machaerium hirtum, Maclura tinctoria, com 4,44\% cada uma e Nectandra oppositifolia, Machaerium villosum, Myrsine umbellata, Casearia decandra, Rollinia sericea e Celtis iguanaea, com $3,3 \%$ cada uma.

Para a nascente 2, observa-se uma densidade média de 2.692 indivíduos.ha 1 , onde as espécies Celtis iguanaea, Nectandra grandiflora, Myrsine umbellata, Casearia sylvestris, Maclura tintoria, Aloysia virgata, Machaerium hirtum, Rollinia sericea, Lonchocarpus muehlbergianus, Fícus guaratinica e Inga striata representam 1884,61 ind.ha $^{-1}$, ou seja $70 \%$ do total. Observa-se que Celtis iguanaea, Nectandra grandiflora, Casearia sylvestris e Maclura tintoria, encontram-se distribuídas em $75 \%$ da nascente 2.

A espécie de maior densidade relativa na nascente 2, foi Celtis iguanae, com $15,71 \%$, seguido pela Nectandra grandiflora, com $8,57 \%$, Myrsine umbellata, com $7,14 \%$ e Casearia sylvestris, Maclura tintoria e Aloysia virgata, com $5,71 \%$ cada uma, as quais juntas correspondem a $48,55 \%$ da densidade total.

Casearia sylvestris, Maclura tintoria, Celtis iguanae e Nectandra grandiflora, apresentaram os maiores valores de frequência relativa, portanto estão melhores distribuídos na nascente 2. Verifica-se no Quadro 2, que a nascente $2(3,051)$ apresentou maior índice de diversidade do que a nascente 1 (2,710). Quanto ao índice de equabilidade de Pielou (J'), observa-se, que a nascente 2 também apresentou o mais alto valor $(91,6 \%)$.

$\mathrm{O}$ fato de a nascente 1 , apresentar menores valores de diversidade e equabilidade, indica a existência de dominância ecológica de algumas espécies no fragmento, ou seja, existe uma maior concentração de indivíduos de espécies dominantes. Este fato evidencia-se pela ocorrência de alta densidade de duas espécies: Lithraea molleoides, com 1.368,42 indivíduos. ha ${ }^{-1}$ e Persea pyrifolia, com 421,05 indivíduos.ha ${ }^{-1}$.

As análises da estrutura da $\mathrm{RN}$ foram realizadas com base nos dados da primeira avaliação. Na nascente 1, no sub-bosque do remanescente, a densidade de indivíduos em regeneração foi estimada em 18.800 indivíduos.ha $^{-1}$ (Quadro 2). Dentre as 51 espécies amostradas, observa-se que, Dendropanax cuneatus (2100 ind.ha ${ }^{-1}$ ), Piper aduncum (1850 ind.ha $\left.{ }^{-1}\right)$, Miconia ibaguensis (1650 ind.ha ${ }^{-1}$ ), ENCICLOPÉDIA BIOSFERA, Centro Científico Conhecer - Goiânia, v.13 n.23; p. 11402016 
Matayba elaeagnoides (1550 ind.ha $\left.\mathrm{h}^{-1}\right)$, nectandra lanceolata $\left(1100\right.$ in.ha $\left.{ }^{-1}\right)$, Chomeli sericea (1000 ind.ha ${ }^{-1}$ ) e Miconia brunnea (1000 ind.ha ${ }^{-1}$ ), foram às espécies que se destacaram, representando, juntas $54,52 \%$ da densidade total da área (Quadro 2).

Como pode ser observado no Quadro 2, dentre as 51 espécies, as seis espécies que apresentaram os maiores valores do parâmetro RN nascente 1. A Figura 1 apresenta a densidade por classe de tamanho da $\mathrm{RN}$, para as seis espécies de maior RN. Observa-se para a nascente 1, no sub-bosque do remanescente, que, durante 0 período estudado, as únicas espécies que apresentaram indivíduos, em todas as classes de tamanho estudadas, foram Dendropanax cuneatus e Piper aduncum, que também apresentaram os maiores valores de RN e Myrsine umbellata. Isso pode ser um indicativo de boas condições para sua regeneração e estabelecimento, pois os resultados sugerem que essas espécies apresentam potencial para se estabelecerem e permanecerem por um período mais longo na floresta.

日P $\square C L$ 四S 目Total

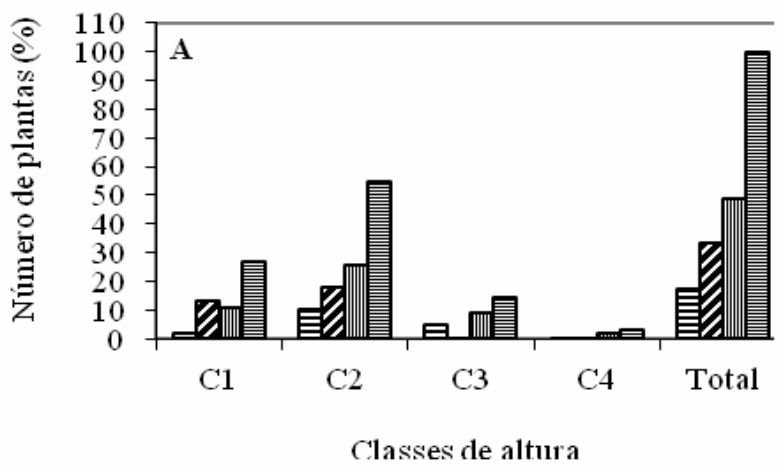

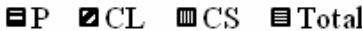

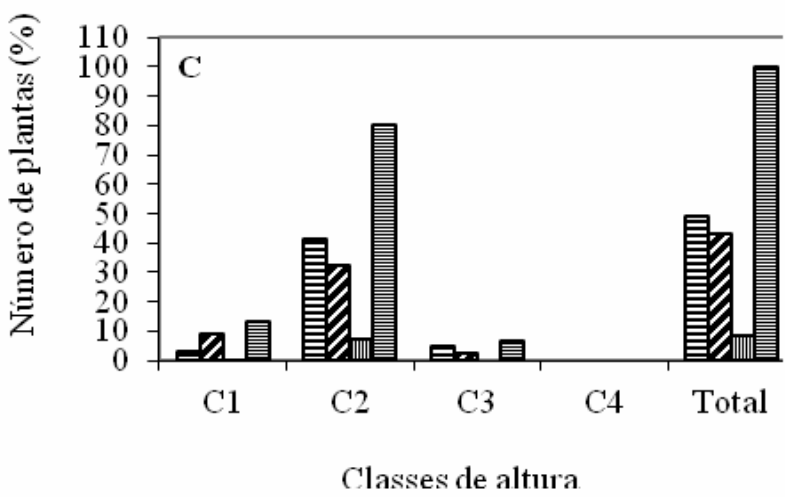

日P $\square \mathrm{CL} \mathbb{\mathrm { UCS }}$ 目Total

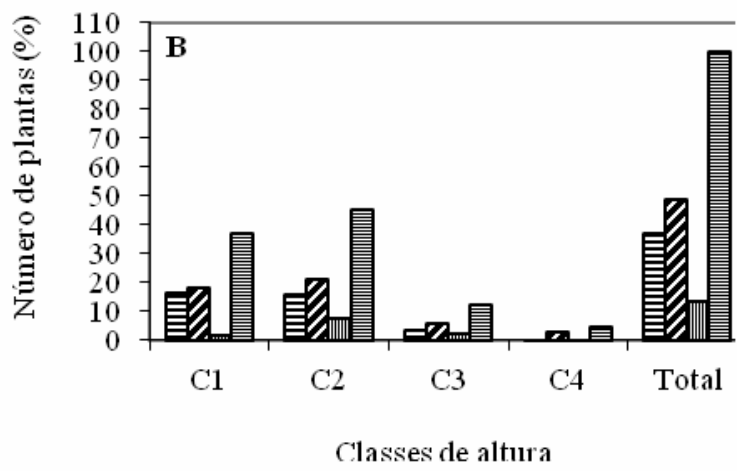

日P $\square \mathrm{CL} \quad \mathbb{U} \mathrm{CS}$ 目Total

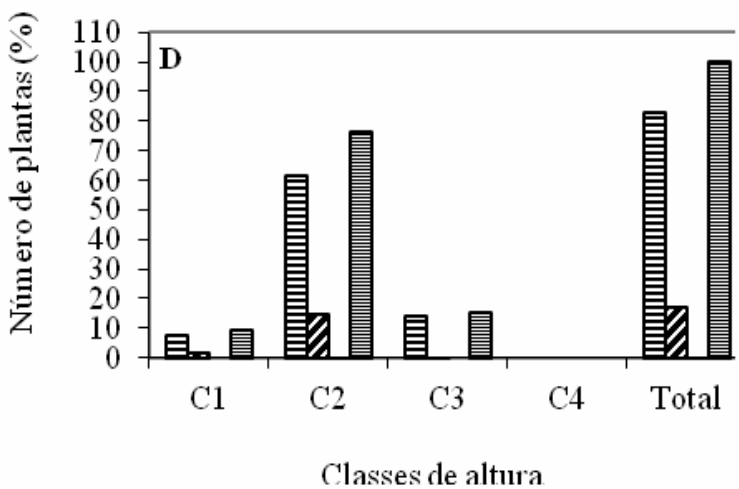

FIGURA 1. Densidade de indivíduos por classe de altura da RN, para as seis espécies de maior regeneração nas matas ciliares no entorno de duas nascentes na cidade de Itumirim, MG. Em que : $P$ = Espécies Pioneiras; $C L=$ Climáx exigente de luz; CS = Climáx tolerantes a sombra; $\mathrm{C} 1$ = Classe de altura 1 (< $30 \mathrm{~cm}) ; \mathrm{C} 2$ = Classe de altura $2(30 \mathrm{a} 150 \mathrm{~cm})$; C3 = Classe de altura $3(150 \mathrm{a}$ $300 \mathrm{~cm}) ; \mathrm{C} 4=$ Classe de altura $4(>300 \mathrm{~cm})$. 
Em relação à similaridade entre áreas para o estrato arbóreo e a $\mathrm{RN}$, na nascente 1, foram encontradas 28 espécies comuns, do total de 76 espécies amostradas. O índice de similaridade de Jaccard para este local foi de $37,0 \%$, indicando que $63,0 \%$ das espécies amostradas não são comuns aos dois estratos. Na nascente 2, área perturbada (AP), de um total de 56 espécies, 19 espécies foram comuns a esses dois estratos. Para esta mesma nascente, o índice de similaridade foi de $34,0 \%$. Para a nascente 2, área degradada (AD), observa-se que, 19 espécies foram comuns aos dois estratos, do total de 60 espécies registradas. $O$ índice de similaridade foi de $32,0 \%$, indicando que $68,0 \%$ das espécies amostradas nesta nascente, não são comuns aos dois estratos avaliados (Quadro 3). Esta baixa similaridade observada entre o estrato arbóreo e a RN, pode ser justificada, por se tratarem de áreas bastante perturbadas, que encontram-se em fase inicial de regeneração.

TABELA 3- Parâmetros fitossociológicos da RN da mata ciliar no entorno de uma nascente do rio Capivari em Itumirim, MG. Em que: DR = densidade relativa (\%);CRTR = classe relativa de tamanho da $\mathrm{RN}, \mathrm{FR}=$ freqüência relativa e $\mathrm{RN}=$ índice de $\mathrm{RN}$.

\begin{tabular}{|c|c|c|c|c|c|c|c|c|}
\hline \multirow[t]{2}{*}{ Espécies } & \multicolumn{4}{|c|}{$\begin{array}{l}\text { Sub-bosque da } \\
\text { nascente } 2\end{array}$} & \multicolumn{4}{|c|}{$\begin{array}{l}\text { Área Alterada da } \\
\text { nascente } 2\end{array}$} \\
\hline & DR & $\mathbf{F R}$ & CRTR & $\mathbf{R N}$ & DR & $\mathbf{F R}$ & CTRR & RN \\
\hline Aegiphila sellowiana & 4.18 & 3.85 & 4.69 & 4.24 & 0.30 & 0.51 & 0.37 & 0.39 \\
\hline Albizia polycephala & 0.84 & 1.92 & 1.05 & 1.27 & 0.90 & 1.01 & 0.46 & 0.79 \\
\hline Aloysia virgata. & & & & & 0.30 & 0.51 & 0.37 & 0.39 \\
\hline Aniba firmula & 0.42 & 0.96 & 0.14 & 0.51 & 13.73 & 13.64 & 12.89 & 13.42 \\
\hline $\begin{array}{l}\text { Aspidosperma } \\
\text { parvifolium }\end{array}$ & & & & & & & & \\
\hline Iymanii & 0.84 & 0.96 & 0.86 & 0.89 & 0.30 & 0.51 & 0.37 & 0.39 \\
\hline Bauhir & & & & & 12.54 & 11.11 & 14.12 & 12.59 \\
\hline Bauhinia Ic & 4.18 & 4.81 & 3.82 & 4.27 & & & & \\
\hline & .11 & 5.77 & 6.27 & 6.39 & 0.30 & 0.51 & 0.37 & 0.39 \\
\hline & 0.42 & 0.96 & 0.43 & 0.60 & & & & \\
\hline Ceik & 4.18 & 5.77 & 4.78 & 4.91 & & & & \\
\hline Celtis & 0.42 & 0.96 & 0.43 & 0.60 & & & & \\
\hline Cestru & & & & & 15.52 & 12.63 & 16.27 & 14.80 \\
\hline Cordia & 1.67 & 3.85 & 2.01 & 2.51 & & & & \\
\hline & & & & & 7.76 & 8.59 & 8.43 & 8.26 \\
\hline Diospyros inconstans & 0.42 & 0.96 & 0.43 & 0.60 & & & & \\
\hline $\begin{array}{l}\text { Erythroxylum } \\
\text { pelleterianum }\end{array}$ & 0.42 & 0.96 & 0.14 & 0.51 & & & & \\
\hline Esenb & 1.26 & 1.92 & 1.11 & 1.43 & & & & \\
\hline & 0.84 & 1.92 & 1.0 & 1.27 & & & & \\
\hline Ilex cerasifolia & 1.26 & 1.92 & 1.30 & 1.49 & & & & \\
\hline Lonchocarpus & & & & & 0.30 & 0.51 & 0.04 & 0.28 \\
\hline Luehea divaricata & 7.11 & 5.77 & 7.42 & 6.77 & 0.30 & 0.51 & & 0.39 \\
\hline Luehea grandiflora & & & & & 0.60 & 1.01 & 0.75 & 0.78 \\
\hline
\end{tabular}




\begin{tabular}{|c|c|c|c|c|c|c|c|c|}
\hline lachaerium nictitans & 1.67 & 3.85 & 2.10 & 2.54 & 0.60 & 1.01 & 0.75 & 0.78 \\
\hline Machaerium villosum & 0.84 & 1.92 & 0.86 & 1.21 & & & & \\
\hline Maclura tinctoria & 1.26 & 1.92 & 1.30 & 1.49 & 0.30 & 0.51 & 0.07 & 0.29 \\
\hline latayba elaeagnoides & 4.18 & 3.85 & 4.21 & 4.08 & 0.60 & 1.01 & 0.75 & 0.78 \\
\hline brunnea & 5.44 & 5.77 & 5.51 & 5.57 & & & & \\
\hline strata & 0.42 & 0.96 & 0.52 & 0.63 & & & & \\
\hline floribunda & 0.42 & 0.96 & 0.52 & 0.63 & & & & \\
\hline diflora & 14.64 & 5.77 & 15.07 & 11.83 & 1.49 & 1.52 & 1.86 & 1.62 \\
\hline eolata & 0.42 & 0.96 & 0.43 & 0.60 & & & & \\
\hline tidula & 0.84 & 1.92 & 0.67 & 1.14 & & & & \\
\hline bosa & 0.84 & 0.96 & 0.67 & 0.82 & & & & \\
\hline Pipe & 0.42 & 0.96 & 0.43 & 0.60 & & & & \\
\hline regnellii & 24.27 & 9.62 & 24.41 & 19.43 & 10.75 & 10.10 & 10.79 & 10.54 \\
\hline egans & 0.42 & 0.96 & 0.43 & 0.60 & & & & \\
\hline escens & & & & & 2.39 & 2.53 & 2.65 & 2.52 \\
\hline Iajava & & & & & 0.30 & 0.51 & 0.37 & 0.39 \\
\hline $\begin{array}{l}\text { syc } \\
\text { arth }\end{array}$ & & & & & 1.49 & 2.02 & 1.21 & 1.57 \\
\hline & 0.84 & 1.92 & 1.05 & 1.27 & & & & \\
\hline & 67 & 0.96 & 0.49 & 1.04 & & & & \\
\hline nthifolius & 0.42 & 0.96 & 0.52 & 0.63 & & & & \\
\hline bana & 0.84 & 1.92 & 1.05 & 1.27 & & & & \\
\hline $\begin{array}{l}n \text { granuloso- } \\
n\end{array}$ & 1.26 & 2.88 & 1.02 & 1.72 & & & & \\
\hline lycocarpum & & & & & 0.90 & 1.01 & & 1.01 \\
\hline Styrax ferrugineus & & & & & 13.13 & 10.10 & 10.08 & 11.10 \\
\hline Vernonanthura diffusa & 0.42 & 0.96 & 0.52 & 0.63 & & & & \\
\hline $\begin{array}{l}\text { nanthura } \\
\text { horica }\end{array}$ & 0.42 & 0.96 & 0.52 & 0.63 & & & & \\
\hline yanthes & & & & & 3.28 & 5.05 & & 3.9 \\
\hline ciliatifolium & 0.42 & 0.96 & 0.43 & 0.60 & 11.34 & 12.63 & 11.32 & 11.76 \\
\hline Zanthoxylum rhoifolium & 1.67 & 3.85 & 1.25 & 2.26 & & & & \\
\hline $\begin{array}{l}\text { Zanthoxylum } \\
\text { xyloperonium. }\end{array}$ & 0.42 & 0.96 & 0.06 & 0.48 & 0.60 & 1.01 & 0.75 & 0.78 \\
\hline t & 100 & & & 100 & 100 & 100 & 100 & 100 \\
\hline
\end{tabular}

Analisando-se todas a nascentes em conjunto, observa-se que, de um total de 97 espécies, 39 são comuns entre o estrato arbóreo e a RN. Dentre as espécies exclusivas da RN observa-se que 19 espécies são exclusivas do sub-bosque do remanescente, e 14 espécies são exclusivas da área em restauração.

\section{CONCLUSÃO}

A nascente 1 apresentou maior riqueza de espécies tanto no estrato arbóreo quanto no estrato regenerante em comparação com a nascente 2. Portanto, faz-se necessário a utilização de técnicas de restauração, combinada com a condução da regeneração natural para que esta nascente recupere o sua função ecológica. 


\section{REFERÊNCIAS}

ALMAZÁN-NUÑEZ, R. C.; ARIZMENDI, M. D. C.; EGUIARTE, L. E.; CORCUERA, P. Changes in composition, diversity and structure of woody plants in successional stages of tropical dry forest in southwest México. Revista Mexicana de Biodiversidad, v. 83, p. 1096-1109, $2012 . \quad$ Disponível em: $<$ http://dx.doi.org/10.7550/rmb.30403> . doi: 10.7550/rmb.30403

CARVALHO, D. A.; OLIVEIRA-FILHO, A. T.; de, VAN DEN BERG, E.; FONTES, M. A. L.; VILELA, E. A.; MARQUES, J. J. G. S. M.; CARVALHO, W. A. C.. Variações florísticas e estruturais do componente arbóreo de uma floresta ombrófila altomontana às margens do rio Grande, Bocaina de Minas, MG, Brasil. Acta Botanica Brasilica, v. 19, n. 1, p. 91-109, 2005. Disponível em: $<$ https://dx.doi.org/10.1590/S0102-33062005000100010>. doi : 10.1590/S010233062005000100010

CASTRO, M. N.; CASTRO, R, M.; SOUZA, P. C. A importância da mata ciliar no contexto da conservação do solo. Revista Eletrônica de Educação da Faculdade Araguaia, v. $4, \quad$ n. $4, \quad 2013 . \quad$ Disponível em: $<$ http://www.faculdadearaguaia.edu.br/sipe/index.php/renefara/article/view/172/156>

FERREIRA, M. J., et al. Avaliação da regeneração natural em nascentes perturbadas no município de Lavras, MG. Ciência Florestal, v. 19, n. 2, p. 109-129, 2009. Disponivel em: <http://dx.doi.org/10.5902/19805098403>. doi: $10.5902 / 19805098403$

GONÇALVES, T. S. Geografia e mecanismos da biodiversidade na regeneração dos fragmentos de floresta estacional decidual da Serra do Cipó em Minas Gerais. Brazilian Geographical Journal: Geosciences and Humanities research medium, v. $6, \quad$ n. 1 , p. 121-139, 2015. Disponível em: $<$ http://www.seer.ufu.br/index.php/braziliangeojournal/article/view/27707/18006>

LOPES, I. S., FELICIANO, A. L. P., MARAGON, L. C., ALENCAR, A. L. DInâmica da regeneração natural no sub-bosque de pinus caribaea morelet. var. caribaea na reserva biológica de saltinho, Tamandaré --PE. Ciência Florestal, v. 26, n. 1, p. 95107, 2016. Disponível em: <http://dx.doi.org/10.5902/1980509821094>. doi: $10.5902 / 1980509821094$

MALLMANN, I. T.; SILVA, V. L.; SCHMITT, J. L. Estrutura comunitária de samambaias em mata ciliar: avaliação em gradiente de antropização. Revista Ambiente \& Água, v. 11, n. 1, p. 110-124, 2016. Disponível em: <http://dx.doi.org/10.4136/ambi-agua.1717 >. doi: 10.4136/ambi-agua.1717

RODRIGUES, R. R. R. Pacto pela restauração da mata atlântica: referencial dos conceitos e ações de restauração florestal. Piracicaba: ESALQ/USP, 2009.

SILVA, R. G., FARIA, R. A. V. B., MOREIRA, L. G., PEREIRA, T. L., SILVA, C.H., BOTELHO, S. A. Avaliação do processo de restauração de área de preservação permanente degradada no sul de Minas Gerais. Revista em Agronegócio e Meio Ambiente, v. 9, n. 1, p. 147-162, 2016. Disponível em: 
<http://dx.doi.org/10.17765/2176-9168.2016v9n1p147-162>. doi: 10.17765/21769168.2016v9n1p147-162

SOUZA, L. M., FARIA, R. A. V. B., BOTELHO, S. A., FONTES, M. A. L., \& FARIA, J. $M$. R. Potencial da regeneração natural como método de restauração do entorno de nascente perturbada. Cerne, v. 18, n. 4, p. 565-576, 2012. Disponível em: $<$ http://dx.doi.org/10.1590/S0104-77602012000400006>. doi : 10.1590/S010477602012000400006 